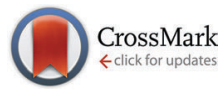

Cite this: Mater. Horiz., 2017, 4,83

Received 11th October 2016, Accepted 19th October 2016

DOI: $10.1039 / c 6 m h 00413$

www.rsc.li/materials-horizons

\title{
Efficient triplet-triplet annihilation upconversion in binary crystalline solids fabricated via solution casting and operated in air $\dagger$
}

\author{
Kenji Kamada, ${ }^{a b}$ Yusuke Sakagami, ${ }^{a b}$ Toshiko Mizokuro, ${ }^{a}$ Yutaka Fujiwara, ${ }^{c}$ \\ Kenji Kobayashi, ${ }^{\star c}$ Kaishi Narushima, ${ }^{d}$ Shuzo Hirata ${ }^{d}$ and Martin Vacha ${ }^{d}$
}

\begin{abstract}
Binary crystalline solids consisting of a sensitizer (Pt-octaethylporphyrin) and an emitter (9,10-diphenylanthracene (DPA) or its alkyl-strapped derivative (C7-sDPA)) were fabricated by solution casting under rapid drying conditions and found to show efficient triplet-triplet annihilation upconversion by the suppression of component segregation. Microspectroscopic studies of individual crystalline particles of the binary solids revealed threshold intensities as low as $5 \mathrm{~mW} \mathrm{~cm}{ }^{-2}$, close to solar levels, and UC quantum yields of $20 \%$ were possible when using C7-sDPA as an emitter, even in air.
\end{abstract}

Triplet-triplet annihilation upconversion (TTA-UC) is an emerging technology for converting low-energy photons into higher-energy ones with light intensities close to that of sunlight ${ }^{1-7}$ which has potential uses in photovoltaic devices ${ }^{8-10}$ and photocatalysis. ${ }^{11,12}$ For these purposes, solid TTA-UC systems are preferable, so a number of efforts have been devoted to the development of solid systems such as doped polymers ${ }^{13,14}$ and gels. ${ }^{15,16}$ In TTA-UC, the photon energy absorbed by a triplet sensitizer molecule is passed onto an emitter molecule via a triplet energy transfer (TET) and a pair of the generated emitter triplets interact with each other via TTA, leading to delayed fluorescence as UC emission. Therefore, these bimolecular processes are key to governing the overall UC quantum yield (QY). In the diluted solid systems of the doped polymer and gel, the bimolecular processes mediated by molecular diffusion are slow and thus limit the overall efficiency.

\footnotetext{
${ }^{a}$ IFMRI, National Institute of Advanced Industrial Science and Technology (AIST), Ikeda, Osaka 563-8577, Japan. E-mail: k.kamada@aist.go.jp

${ }^{b}$ Department of Chemistry, School of Science and Technology, Kwansei Gakuin University, Sanda, Hyogo 669-1337, Japan

${ }^{c}$ Department of Chemistry, Faculty of Science, Shizuoka University, Shizuoka 422-8529, Japan. E-mail: kobayashi.kenji.a@shizuoka.ac.jp

${ }^{d}$ Department of Organic and Polymeric Materials, Tokyo Institute of Technology,

Meguro-ku, Tokyo 152-8552, Japan

$\dagger$ Electronic supplementary information (ESI) available: Theoretical methodology, sample preparation methods, details of spectroscopic characterization methods using microscopes, high-resolution spectrographs, quantum yield measurements with integration spheres, absorption and emission spectra of the compounds, polarization and SEM images, upconversion decay data, and additional threshold data for the upconversion. See DOI: 10.1039/c6mh00413j
}

\begin{abstract}
Conceptual insights
This work provides a new concept to realize binary solids that show efficient triplet-triplet annihilation photon upconversion (TTA-UC) via solution casing under rapid drying conditions. It prevents the segregation of the sensitizer molecules on crystallization and provides favorable distribution in the emitter matrix, allowing efficient energy transfer from the sensitizer to the emitter, which is a key step of TTA-UC. This strategy is effective even for a sensitizer-emitter pair that shows no TTA-UC in the crystal due to the segregation (Pt-octaethylporphyrin and 9,10-diphenylanthracene (DPA), in PCCP, 2012, 14, 4322). The conceptual advancement here over the previous approaches for solid state TTA-UCs, such as gels and metal organic frameworks, is its simplicity: no specially designed chromophore with costly synthetic steps is required, showing its wide applicability. Further potential of the concept is shown by using the DPA derivative as an emitter, resulting in a much lower threshold and a high UC quantum yield comparable to those of degassed solution systems. Furthermore, the concept of rapid drying using nonequilibrium may be applicable to other solution processes.
\end{abstract}

Very recently, TTA-UC in dense-chromophore media such as chromophore liquids, ${ }^{17}$ metal-organic frameworks (MOFs), ${ }^{18}$ and deposited bilayers ${ }^{19,20}$ has been reported. In these systems, the migration of triplet excitons mediates the bimolecular processes instead of molecular diffusion, allowing the realization of fast and efficient bimolecular processes.

On the other hand, binary crystals can be an ideal medium for solid TTA-UC because the speed of migration in a crystal can be higher than that in other dense media thanks to the crystal regularity and dense packing. However, TTA-UC in binary solids was reported to be unobservable due to macroscopic segregation of the sensitizer from the emitter matrix ${ }^{5,21}$ leading to poor efficiency of the TET and dimeric quenching of the sensitizer. There are a few reports on TTA-UC in binary solids $;{ }^{2-24}$ however, they require high excitation intensities in the order of $\mathrm{kW} \mathrm{cm}^{-2}$, far greater than the solar range of intensity needed for practical applications.

Herein, we show that a nonequilibrium preparation method can overcome the segregation and realize high upconversion quantum yields (UC-QYs) in binary solids. The key idea is to 
a)
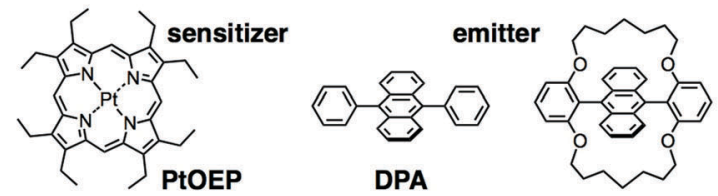

C7-sDPA
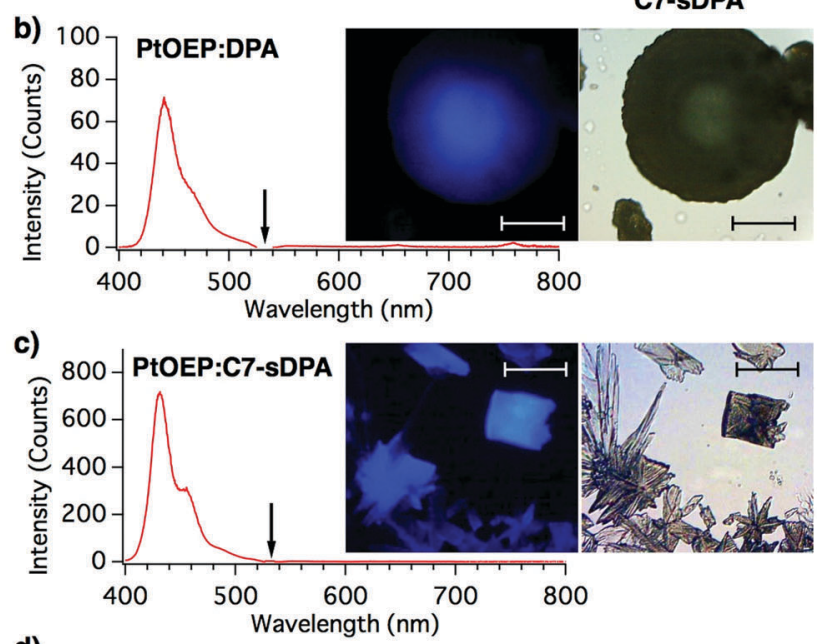

d)

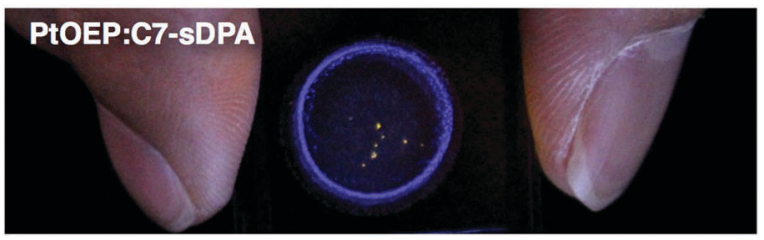

Fig. 1 (a) Chemical structures of the compounds. Upconverted emission spectra and microphotographs (insets: emission (left) and transmission (right) images) of binary crystals of (b) PtOEP:DPA and (c) PtOEP:C7-sDPA fabricated via drop casting under rapid-drying conditions. The excitation wavelength $(532 \mathrm{~nm})$ is shown by the arrows. All scale bars represent $50 \mu \mathrm{m}$. (d) Centimeter-scale upconverted emission image of the PtOEP: C7-sDPA cast sample under illumination from an expanded beam from a diode laser ( $532 \mathrm{~nm}$, excitation intensity: $\sim 1 \mathrm{~mW} \mathrm{~cm}^{-2}$ ) through a notch filter The crystalline particles formed in the ring-like region show upconversion.

form an emitter matrix via rapid crystallization before the sensitizer segregates. To achieve this goal, we adopted a simple method, i.e. drop casting of the solution under rapid drying conditions. Two different emitter molecules, 9,10-diphenylanthracene (DPA) and its derivative, C7-sDPA, were used with a common sensitizer, Pt-octaethylporphyrin (PtOEP), as shown in Fig. 1a. Both DPA and C7-sDPA have fluorescence (FL) QY close to unity ( $\Phi_{\mathrm{f}}=0.95$ and 0.85 , respectively) in crystals, ${ }^{25}$ making them suitable for use as emitters for TTA-UC in the solid state. The PtOEP/DPA system has been used as a benchmark in many works in solution, ${ }^{11,22-24,26-28}$ and has also been used in previous studies of binary solids. ${ }^{29-31}$ Additionally, C7-sDPA shows excellent photostability compared to DPA and invariant FL properties regardless of its morphology in the solid state ${ }^{25}$ because of the loop-like alkoxy chain protecting the anthracene core. In this work, we show that these alkoxy chains provide a positive effect on the compatibility of the material with PtOEP, thus enabling uniform dispersion of the sensitizer in the emitter matrix.

Binary crystals of PtOEP:DPA and PtOEP:C7-SDPA were fabricated by drop casting on a slide glass (ESI $\dagger$ ) from mixed tetrahydrofuran (THF) solutions of PtOEP and the respective emitters. The concentrations of the respective emitters were set at their saturated concentrations (143 mM for DPA and $21 \mathrm{mM}$ for C7-sDPA). The molar ratios of PtOEP to the emitters were $1: 1000$ for DPA and 1:738 for C7-sDPA unless otherwise noted, but a ratio of 1:1000 for C7-sDPA was also prepared (see below). THF was chosen as the solvent because of its low boiling point and good wettability on glass substrates, as well as the high solubility of the emitters in it. THF spread widely on glass and dried quickly, and gave the best results of the several common solvents tested. The crystals precipitated from the periphery of the drop during drying and formed a ring-shaped rim that took on the appearance of a coffee stain.

The rim of the cast film of PtOEP:DPA was found to consist of many dark-colored round particles (10-100 $\mu \mathrm{m}$ in size, Fig. 1b), which showed spherulite-like structures when observed using polarized microscopy (Fig. S7 in the ESI $\dagger$ ). SEM images showed that the particles consisted of aggregations of needle-like microcrystals with widths of $\sim 1 \mu \mathrm{m}$ (Fig. S8, ESI $\dagger$ ). The particles were found to show a clear blue emission under continuous wave (cw)-light irradiation at $532 \mathrm{~nm}$, which is the wavelength of the Q-band of PtOEP, from a laser diode under an optical microscope. The $532 \mathrm{~nm}$ excitation was confirmed to induce a blue emission by placing a shortcut filter (Y44) in the excitation path. The emission spectrum from the particle (with a peak at $440 \mathrm{~nm}$ ) matched that of the fluorescence of DPA in the powdered form (Fig. S5, ESI $\dagger$ ). From these results, the blue emission from the particle was assigned to the UC emission. The monomeric $(650 \mathrm{~nm})$ and dimeric $(780 \mathrm{~nm})^{23}$ phosphorescence emission peaks of PtOEP (Fig. S4, ESI $\dagger$ ) were negligible in the spectrum, suggesting that efficient TET occurred from the sensitizer to the DPA matrix, unlike in previous cases with crystals ${ }^{5}$ and spincoated films, ${ }^{23}$ where the UC emission and phosphorescence peaks were of similar intensities. The excitation intensity at the sample position $\left(4 \mathrm{~W} \mathrm{~cm}^{-2}\right)$ was three orders of magnitude lower than that reported for the same binary solid. ${ }^{22,23}$

In the case of PtOEP:C7-sDPA, rectangular- and petal-shaped crystals were found in the rim. These crystalline particles also showed blue UC emission when excited at $532 \mathrm{~nm}$ (Fig. 1c). The emission spectrum matched that of the fluorescence of C7-sDPA in the powder form. Not all particles were luminescent, but drop casting led to the formation of many luminescent particles in a reproducible manner. The UC emission of PtOEP:C7-sDPA was stronger than that of PtOEP:DPA and was successfully observed by centimeter-scale wide-area irradiation (Fig. 1d). The ring-like pattern of the UC emission under illumination with the expanded beam $(2 \mathrm{~cm}$ in diameter) of the laser diode $(4 \mathrm{~mW})$ corresponded to the rim where the luminescent particles had formed. The excitation intensity was $1 \mathrm{~mW} \mathrm{~cm}^{-2}$, demonstrating that TTA-UC occurred at excitation intensities comparable to those in sunlight $\left(\sim 1 \mathrm{~mW} \mathrm{~cm}{ }^{-2}\right.$ for $532 \pm 5 \mathrm{~nm})^{26}$

To obtain quantitative information on the UC properties, we measured the excitation intensity $\left(I_{\mathrm{ex}}\right)$ dependence of the UC-emission for individual particles under an $\mathrm{Ar}$ atmosphere (Fig. 2a). The dependence was quadratic at low excitation 

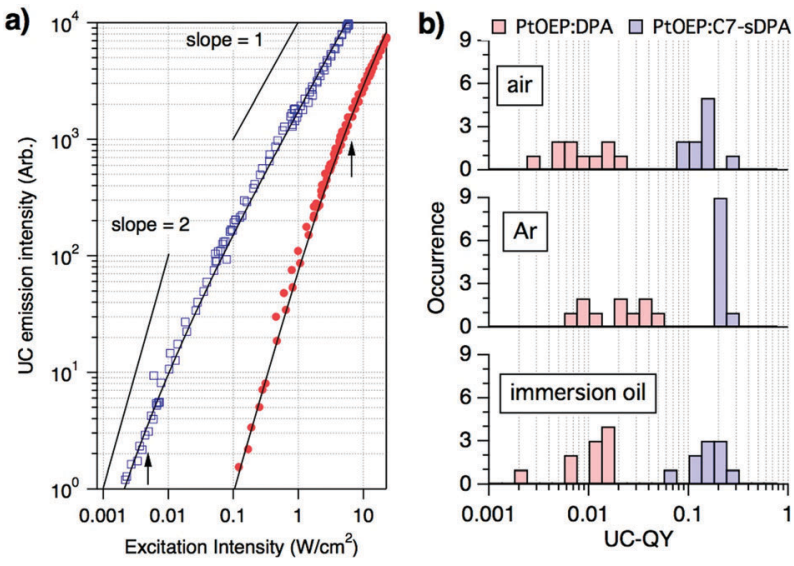

Fig. 2 (a) Examples of the excitation intensity dependence of the upconverted emission from single microparticles of PtOEP:DPA (filled circle) and PtOEP:C7-sDPA (open square) in Ar, with the respective curves being fitted with eqn (1) (solid curves). The arrows indicate the positions of the obtained threshold intensities from the curve fits $\left(I_{\text {th }}=6.8 \pm 2.8 \mathrm{~W} \mathrm{~cm}^{-2}\right.$ for PtOEP:DPA and $4.6 \pm 2.2 \mathrm{~mW} \mathrm{~cm} \mathrm{~cm}^{-2}$ for PtOEP:C7-sDPA). More data can be found in the ESI. $\dagger$ (b) Histograms of the upconversion quantum yields (UC-QY) of individual microparticles of PtOEP:DPA and PtOEP:C7-sDPA in different environments.

intensities but gradually changed to a linear dependence with increasing intensity. This behavior is characteristic of TTA-UC and is explained by a transition from a second order reaction to a quasi-first order one for TTA between the emitter triplets as their concentration increases. ${ }^{5}$ The observed dependences could be accurately reproduced using the theoretical equation: ${ }^{32}$

$$
I_{\mathrm{UC}}=K\left[1+\left(1-\sqrt{4 I_{\mathrm{ex}} / I_{\mathrm{th}}}\right) /\left(2 I_{\mathrm{ex}} / I_{\mathrm{th}}\right)\right] I_{\mathrm{ex}}
$$

derived from the standard kinetic model of TTA-UC (see the ESI $\dagger$ ). Here $K$ is the proportionality constant and $I_{\text {th }}$ is the threshold intensity, a sensitivity measure of the TTA-UC system, defined as the excitation intensity at the crossing point between the extrapolated lines of the quadratic and linear dependences. One can obtain $I_{\text {th }}$ in a more reproducible manner by fitting the curve with eqn (1) than by extrapolation of the two slopes on the double logarithmic plot. The obtained $I_{\text {th }}$ fluctuated from one particle to another but ranged from 0.2 to $7 \mathrm{~W} \mathrm{~cm}{ }^{-2}$ for DPA and from 5 to $24 \mathrm{~mW} \mathrm{~cm}^{-2}$ for C7-sDPA (Fig. 2a and Fig. $\mathrm{S} 10, \mathrm{~S} 11, \mathrm{ESI} \dagger)$. The $I_{\mathrm{th}}$ value of C7-sDPA was lower by nearly 2 orders of magnitude than that of DPA. These $\mathrm{mW} \mathrm{cm}^{-2}$-order values for $I_{\text {th }}$ in PtOEP:C7-sDPA are of the same order as that of sunlight, and are consistent with the centimeter-scale demonstration in Fig. 1d.

UC-QY is also an important parameter for the characterization of TTA-UC systems. We therefore evaluated the UC-QY of individual particles by measuring the absorption and emission spectra of the same particle under a microscope (see the ESI $\dagger$ for the methodological details). Here, full conversion is defined as an UC-QY of $100 \%$ (eqn (S9), ESI $\dagger$ ). The data were collected for 10 particles under different environments (air, Ar, and immersion oil). The immersion oil was used to check the effect of scattering on the measurements because it reduces the refractive-index mismatch between the environment and the particle. The obtained UC-QYs varied depending on the particle; however, different environments did not show large differences beyond the distribution of the particles (Fig. 2b). The values in Ar and in air were almost the same, suggesting that oxygen cannot penetrate the particles. The values in the immersion oil showed that the effect of refractive index mismatching was negligible. Scattering by the particle can be a source of considerable error in absorption measurements. However, the error was less than $10 \%$ for the absorbance at the excitation wavelength after subtraction of the baseline (in the ESI $\dagger$ ), and the resulting error in QY was again within the range of the distribution. On the other hand, significant differences were seen between the emitters. The UC-QY of PtOEP:DPA showed wide distributions centered at around 1-3\% regardless of the environment. A separate, complementary UC-QY measurement carried out using an integration sphere (ESI $\dagger$ ) gave a spatially averaged UC-QY of 3\% for the same sample of PtOEP:DPA. This excellent agreement shows the reliability of our microscopic measurements. On the other hand, the UC-QY of PtOEP:C7-sDPA was distributed at around 10-20\%, nearly ten times higher than that of PtOEP:DPA. We also repeated the same measurement for the PtOEP:C7-sDPA sample with a molar ratio of $1: 1000$, but the obtained result was almost identical (Fig. S12, ESI $\dagger$ ). The cast samples showed long-term stability; similar orders of UC-QYs were obtained for the PtOEP:DPA and PtOEP:C7-sDPA samples stored for 11 months under an Ar atmosphere.

The highest UC-QY reported thus far for binary solid systems is $0.3 \%$ in a deposited bilayer, ${ }^{20}$ and $4 \%$ (rescaled to the full conversion of $100 \%$ ) in a MOF. ${ }^{18}$ Very recently, UC in a binary crystal also fabricated using a casting method has been reported. ${ }^{33}$ The UC-QY value reported was $0.20-0.28 \%$ (also rescaled to $100 \%$ ) using 9-alkoxy-substituted 10-phenylanthracene as an emitter. ${ }^{33}$ Compared to these reported values, the UC-QY of PtOEP:C7-sDPA is considered to be a record high value in binary solids. The UC-QYs obtained are comparable to those of solution systems of PtOEP and DPA, although these values are varied, ranging from $5 \%$ in dimethyl formamide ${ }^{34}$ to $46 \%$ (rescaled to $100 \%)$ in $\mathrm{THF}^{14}$

To understand the reason for the lower $I_{\text {th }}$ and higher UC-QY of PtOEP:C7-sDPA compared to PtOEP:DPA, we focused our attention on the absorption peak of the Q-band of PtOEP. For PtOEP:C7-sDPA (Fig. 3a), the Q-band (at $\lambda_{\mathrm{abs}}=535 \mathrm{~nm}$, bandwidth of $\Delta \lambda=13 \mathrm{~nm}$ as calculated from the full width at half maximum) was almost identical to that in solution $\left(\lambda_{\mathrm{abs}}=\right.$ $534 \mathrm{~nm}, \Delta \lambda=11 \mathrm{~nm}$ in DMSO). Meanwhile, for PtOEP:DPA (Fig. 3b), the Q-band was broadened and red-shifted $\left(\lambda_{\mathrm{abs}}=\right.$ $542 \mathrm{~nm}, \Delta \lambda=27 \mathrm{~nm}$ ), as seen in aggregated forms of PtOEP such as the PtOEP nanocrystals prepared via precipitation $\left(\lambda_{\mathrm{abs}}=541 \mathrm{~nm}, \Delta \lambda=18 \mathrm{~nm}\right)$. These results suggest that PtOEP was well dispersed in the C7-sDPA matrix, resulting in a solutionlike environment, but formed aggregates in the DPA matrix.

Observation of high-resolution microscopic spectrographs at the UC wavelengths (400-480 nm, with a $100 \times$ objective lens) supported the difference in the distribution of PtOEP in the matrices: the UC emission was uniform over the particle for 
a)

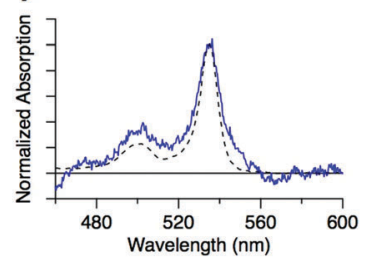

c)

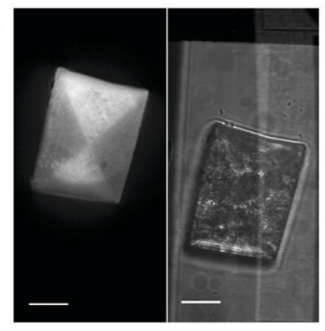

b)

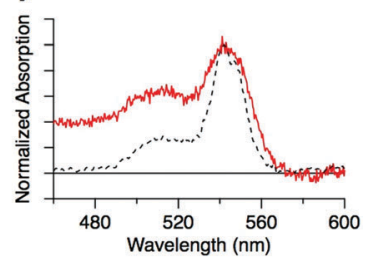

d)

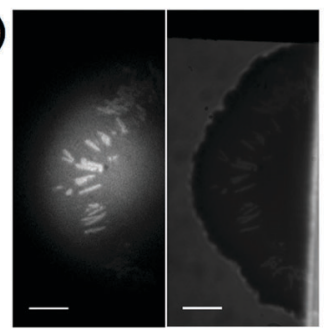

Fig. 3 (a) Absorption spectra of the Q-band of PtOEP in binary crystals of PtOEP:C7-sDPA (solid line) and in dimethyl sulfoxide solution (dashed line). (b) Absorption spectra of the Q-band in binary crystals of PtOEP:DPA (solid line) and of PtOEP nanocrystals dispersed in water prepared using the precipitation method (dashed line). High magnification spectrographic image of the upconverted emission (left) and transmission image (right) of the (c) PtOEP:C7-sDPA and (d) PtOEP:DPA particles. All scale bars represent $10 \mu \mathrm{m}$.

PtOEP:C7-sDPA (Fig. 3c), while for PtOEP:DPA, most of the UC emission occurred from rod-like substructures (Fig. 3d). This suggests that PtOEP was distributed uniformly in the C7-sDPA matrix while it aggregated in DPA. The improved dispersion accelerates the TET and results in a higher TET QY $\left(\Phi_{\text {TET }}\right)$. Thus, the higher $\Phi_{\text {TET }}$ leads to a higher UC-QY as well as a lower $I_{\text {th }}$ due to the equation $I_{\text {th }}=\left(\alpha \Phi_{\text {TET }} k_{\mathrm{TTA}} \tau_{\mathrm{TE}}^{2}\right)^{-1},{ }^{26}$ where $\alpha$ is the absorption coefficient, $k_{\mathrm{TTA}}$ is the second-order rate constant of the TTA process, and $\tau_{\mathrm{TE}}$ is the triplet lifetime of the emitter. This relationship shows that $\tau_{\mathrm{TE}}$ is another factor that determines $I_{\mathrm{th}}$. UC-emission decay measurements were performed on the binary solids (data shown in Fig. S9, ESI $\dagger$ ), and we obtained $\tau_{\mathrm{TE}}$ values of $140 \mu \mathrm{s}$ for C7-sDPA and $54 \mu \mathrm{s}$ for DPA. The longer $\tau_{\mathrm{TE}}$ of C7-sDPA could also result in a lower $I_{\text {th }}$ compared to DPA.

To obtain more information on the distribution of PtOEP, we performed scanning TEM observations on the particles of

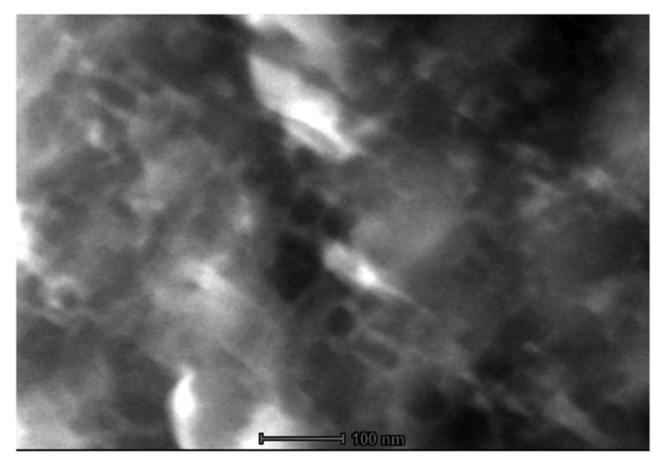

Fig. 4 High-angle annular dark field imaging in the scanning transmission electron microscopy (HAADF/STEM) image of a crystalline particle of PtOEP:C7-sDPA. The scale bar represents $100 \mathrm{~nm}$.

PtOEP:C7-sDPA. Although the distribution is considered to be uniform from the absorption spectrum and the high-resolution microscopic spectrograph as mentioned above, the PtOEP: C7-sDPA particle was found to have a cellular structure (Fig. 4) at the nanometer scale. In these images, only the heavy Pt atom of PtOEP gave bright areas, so the bright boundary-like cell walls corresponded to the distribution of PtOEP. This result shows that PtOEP was not truly molecularly dispersed at the nanometer scale, and was instead localized to surround nano-cells of C7-sDPA. The size of the nanostructure was 20-50 nm, shorter than the triplet diffusion length $\left(L_{\mathrm{d}} \sim 230 \mathrm{~nm}\right.$ or longer $)$ estimated from the $\tau_{\mathrm{TE}}$ of C7-SDPA and the diffusion coefficient $\left(D=4 \times 10^{-6} \mathrm{~cm}^{2} \mathrm{~s}^{-1}\right.$ or larger, calculated from $\left.I_{\mathrm{th}} \sim 10 \mathrm{~mW} \mathrm{~cm}^{-2}\right)$ using the relation $L_{\mathrm{d}}=\left(D \tau_{\mathrm{TE}}\right)^{1 / 2}$. Thus, it is considered that a triplet exciton of C7-sDPA generated at the boundary by TET could migrate to the other end of the nano cell. This enhances the probability of TTA and also contributes to the high UC-QY.

\section{Conclusions}

In conclusion, we demonstrated efficient TTA-UC in binary solids fabricated via rapid-drying solution casting, even with the benchmark pairing of PtOEP and DPA. Compared to DPA, $I_{\text {th }}$ and UC-QY were improved when the alkoxy-strapped derivative C7-sDPA was used, which allows centimeter-scale large-area UC emission for excitation at intensities close to those of sunlight and high UC-QYs of up to $20 \%$ even in air. The compatibility of C7-sDPA gave a better distribution of the sensitizer on the $\mu \mathrm{m}$ scale. The low $I_{\text {th }}$ and high UC-QY are considered to originate from the non-aggregated distribution of the sensitizer and the long triplet lifetime. The nanostructure found through TEM observations also contributed to UC-QY through efficient TTA. It should be emphasized that the results show the great potential of binary solid systems. The use of nonequilibrium processes to realize favorable dispersions in binary systems is not limited to the casting method presented here, but could be applied to other techniques such as spray drying or ink-jet printing.

\section{Acknowledgements}

This work was supported by JSPS KAKENHI Grant Numbers JP26107004 and JP26107014 from Scientific Research on Innovative Areas "Photosynergetics". We thank Dr Naoyuki Kitamura of AIST for his help in acquiring the SEM images and sincerely thank Dr Takeyuki Uchida and Shin Horiuchi of Tsukuba Innovation Arena, AIST, for the HAADF/STEM observations.

\section{Notes and references}

1 D. V. Kozlov and F. N. Castellano, Chem. Commun., 2004, 2860-2861.

2 R. R. Islangulov, D. V. Kozlov and F. N. Castellano, Chem. Commun., 2005, 3776-3778.

3 S. Baluschev, T. Miteva, V. Yakutkin, G. Nelles, A. Yasuda and G. Wegner, Phys. Rev. Lett., 2006, 97, 143903.

4 P. Ceroni, Chem. - Eur. J., 2011, 17, 9560-9564. 
5 A. Monguzzi, R. Tubino, S. Hoseinkhani, M. Campione and F. Meinardi, Phys. Chem. Chem. Phys., 2012, 14, 4322-4332.

6 J. Zhou, Q. Liu, W. Feng, Y. Sun and F. Li, Chem. Rev., 2015, 115, 395-465.

7 Y. Y. Cheng, B. Fückel, T. Khoury, R. l. G. C. R. Clady, M. J. Y. Tayebjee, N. J. Ekins-Daukes, M. J. Crossley and T. W. Schmidt, J. Phys. Chem. Lett., 2010, 1, 1795-1799.

8 A. Nattestad, Y. Y. Cheng, R. W. Macqueen, T. F. Schulze, F. W. Thompson, A. J. Mozer, B. Fu, T. Khoury, M. J. Crossley, K. Lips, G. G. Wallace and T. W. Schmidt, J. Phys. Chem. Lett., 2013, 4, 2073-2078.

9 C. Simpson, T. M. Clarke, R. W. MacQueen, Y. Y. Cheng, A. J. Trevitt, A. J. Mozer, P. Wagner, T. W. Schmidt and A. Nattestad, Phys. Chem. Chem. Phys., 2015, 17, 24826-24830.

10 A. Monguzzi, D. Braga, M. Gandini, V. C. Holmberg, D. K. Kim, A. Sahu, D. J. Norris and F. Meinardi, Nano Lett., 2014, 14, 6644-6650.

11 J.-H. Kim and J.-H. Kim, J. Am. Chem. Soc., 2012, 134, 17478-17481.

12 O. S. Kwon, J.-H. Kim, J. K. Cho and J.-H. Kim, ACS Appl. Mater. Interfaces, 2015, 7, 318-325.

13 R. R. Islangulov, J. Lott, C. Weder and F. N. Castellano, J. Am. Chem. Soc., 2007, 129, 12652-12653.

14 A. Monguzzi, F. Bianchi, A. Bianchi, M. Mauri, R. Simonutti, R. Ruffo, R. Tubino and F. Meinardi, Adv. Energy Mater, 2013, 3, 680-686.

15 R. Vadrucci, C. Weder and Y. C. Simon, Mater. Horiz., 2015, 2, 120-124.

16 P. Duan, N. Yanai, H. Nagatomi and N. Kimizuka, J. Am. Chem. Soc., 2015, 137, 1887-1894.

17 P. Duan, N. Yanai and N. Kimizuka, J. Am. Chem. Soc., 2013, 135, 19056-19059.
18 P. Mahato, A. Monguzzi, N. Yanai, T. Yamada and N. Kimizuka, Nat. Mater., 2015, 14, 924-930.

19 M. Wu, D. N. Congreve, M. W. B. Wilson, J. Jean, N. Geva, M. Welborn, T. Van Voorhis, V. Bulovié, M. G. Bawendi and M. A. Baldo, Nat. Photonics, 2015, 10, 31-34.

20 T. C. Wu, D. N. Congreve and M. A. Baldo, Appl. Phys. Lett., 2015, 107, 031103.

21 M. Campione, E. Fumagalli, L. Raimondo, A. Monguzzi, F. Meinardi and A. Sassella, Chem. Mater., 2011, 23, 832-840.

22 S. Baluschev, V. Yakutkin, G. Wegner, B. Minch, T. Miteva, G. Nelles and a. Yasuda, J. Appl. Phys., 2007, 101, 023101.

23 H. Goudarzi and P. E. Keivanidis, J. Phys. Chem. C, 2014, 118, 14256-14265.

24 R. Karpicz, S. Puzinas, V. Gulbinas, A. Vakhnin, A. Kadashchuk and B. P. Rand, Chem. Phys., 2014, 429, 57-62.

25 Y. Fujiwara, R. Ozawa, D. Onuma, K. Suzuki, K. Yoza and K. Kobayashi, J. Org. Chem., 2013, 78, 2206-2212.

26 A. Monguzzi, J. Mezyk, F. Scotognella, R. Tubino and F. Meinardi, Phys. Rev. B: Condens. Matter Mater. Phys., 2008, 78, 195112.

27 A. Monguzzi, M. Frigoli, C. Larpent, R. Tubino and F. Meinardi, Adv. Funct. Mater., 2012, 22, 139-143.

28 A. Haefele, J. Blumhoff, R. S. Khnayzer and F. N. Castellano, J. Phys. Chem. Lett., 2012, 3, 299-303.

29 H. Sternlicht, G. C. Nieman and G. W. Robinson, J. Chem. Phys., 1963, 38, 1326-1335.

30 D. Y. Kondakov, J. Soc. Inf. Disp., 2009, 17, 137-144.

31 H. Fukagawa, T. Shimizu, N. Ohbe, S. Tokito, K. Tokumaru and H. Fujikake, Org. Electron., 2012, 13, 1197-1203.

32 Y. Murakami, Chem. Phys. Lett., 2011, 516, 56-61.

33 M. Hosoyamada, N. Yanai, T. Ogawa and N. Kimizuka, Chem. - Eur. J., 2016, 22, 2060-2067.

34 X. Cao, B. Hu and P. Zhang, J. Phys. Chem. Lett., 2013, 4, 2334-2338. 